PALEO

Revue d'archéologie préhistorique

$14 \mid 2002$

Varia

\title{
À propos des burins du Raysse du Flageolet I
} (Dordogne, France)

About the Raysse burins of the Flageolet I (Dordogne, France)

\section{Geraldine Lucas}

\section{OpenEdition}

Journals

Édition électronique

URL : http://journals.openedition.org/paleo/1397

DOI : $10.4000 /$ paleo. 1397

ISSN : 2101-0420

Éditeur

SAMRA

Édition imprimée

Date de publication : 1 décembre 2002

Pagination : 63-76

ISSN : 1145-3370

\section{Référence électronique}

Geraldine Lucas, « À propos des burins du Raysse du Flageolet । (Dordogne, France) », PALEO [En

ligne], 14 | 2002, mis en ligne le 10 août 2010, consulté le 26 juillet 2020. URL : http://

journals.openedition.org/paleo/1397 ; DOI : https://doi.org/10.4000/paleo.1397

Ce document a été généré automatiquement le 26 juillet 2020.

\section{c) (i) $९$}

PALEO est mis à disposition selon les termes de la licence Creative Commons Attribution - Pas d'Utilisation Commerciale - Pas de Modification 4.0 International. 


\title{
À propos des burins du Raysse du Flageolet I (Dordogne, France)
}

\author{
About the Raysse burins of the Flageolet I (Dordogne, France)
}

Geraldine Lucas

Je tiens à remercier ici Jean-Philippe Rigaud qui m'a confié ce travail et encadré dans son accomplissement, Maureen A. Hays dont la collaboration est toujours d'un grand intérêt scientifique, Laurent Klaric qui a eu la gentillesse de relire cet article et de me faire parvenir ses travaux encore non-publiés, Pierre Bodu et Michel Lenoir qui ont également pris le temps de relire ce travail et de me transmettre leurs remarques et tous les fouilleurs du Flageolet I sans qui aucune étude n'aurait été possible.

\section{Introduction}

1 Les burins du Raysse (ou de Bassaler) ont suscité, dans les années 1970, un intérêt particulier de la part de L. Pradel (Pradel, 1953, 1965, 1966a, 1966b, 1971, 1977, Pradel et Pradel, 1966) et de H.-L. Movius et N.-C. David (Movius etDavid, 1970) afin de préciser leur position chronologique et de caractériser leur mode de fabrication. A Pataud, comme au Flageolet I (Rigaud, 1982) et aux Jambes (Célerier 1967), les burins du Raysse semblent liés à une forte diminution des burins de Noailles (Movius et David, 1970).

Deux récentes études ont montré, par une approche technologique, qu'une des fonctions très probables de cet «outil», et qui n'avait pas été envisagée par ces précédents auteurs, est celle de nucléus à lamelles (Le Mignot, 2000, Klaric, 1999, 2000, Klaric et al. 2001). Le travail de Laurent Klaric est particulièrement intéressant puisqu'il décrit plusieurs modalités de production lamellaire associées sur un même "nucléusburin du Raysse ». La première d'entre elles est l'exploitation de la face supérieure d'une lame aménagée par la technique de Kostienki depuis un plan de frappe formé par une troncature inverse (Klaric, 2000). Cette modalité est associée presque systématiquement au débitage sur bord de lame ou "technique de coup de burin" (seconde variante). La troisième, associée également aux deux premières, implique 
l'extraction des lamelles sur la face inférieure du support (telle que l'on peut l'observer sur les burins du Raysse). Parallèlement à ces études, nous avons fait une observation similaire sur les burins du Raysse de la couche V du Flageolet I (fig. 1) (Lucas, 2000) ${ }^{1}$ aboutissant à cette même conclusion. Nous proposons de donner ici les raisons qui ont conduit à cette réflexion sans omettre d'évoquer la possibilité d'une réutilisation de ces burins comme outils (Hays et Lucas, 2000a).

Fig. 1 - Burins du Raysse, couche V, Flageolet I

Fig. 1 - Raysse burins, level V, Flageolet I

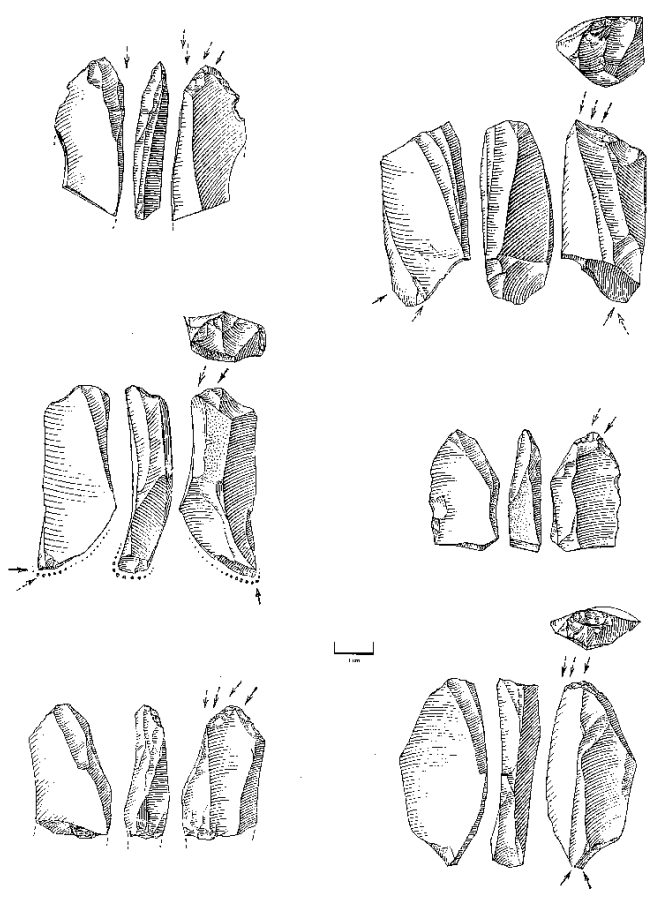

Ces burins présentent des degrés d'exploitation différents repérables à la forme de la troncature : plus l'exploitation est avancée, plus la troncature formera un angle ( $n^{\circ} 1,4$ et 5 ).

These burins illustrate different degrees of exploitation as observed by the shape of the truncation: the more advanced the exploitation, the more angular the truncation ( $n^{\circ} 1,4$ and 5$)$.

(dessins de J.-G. Marcillaud). (drawings by J.-G. Marcillaud).

Le matériel étudié provient de l'abri du Flageolet I situé en Dordogne (fig. 2). Le site fut découvert et fouillé par Jean-Philippe Rigaud entre 1967 et 1984. L'archéostratigraphie présente une importante séquence aurignaco-périgordienne s'étalant entre $18610 \pm 440$ (Ly 2185) et $33800 \pm 1800$ (OxA 598) (fig. 3). Le niveau étudié, la couche V, est daté à $25700 \pm 700$ (OxA 447) (Rigaud, 1993 et Mellars et al., 1987). L'ensemble lithique comprend 6507 objets (refus de tamisage, soit $3832 \mathrm{~g}$ de matériel lithique, exclus) dont 546 outils (soit $8 \%$ ). Parmi le faible nombre de pièces à dos, trois fragments de pointes de la Gravette sont présentes (celles-ci sont fabriquées sur de petits fragments de lame). Les outils caractéristiques du Périgordien $\mathrm{V}$ de Peyrony sont peu nombreux : aucune pointe de la Font-Robert, trois éléments tronqués et onze burins de Noailles. En revanche, les burins de toutes sortes, dont les burins du Raysse présents au Flageolet I dans ce niveau $(\mathrm{n}=36)$, sont abondants (Rigaud, 1982, Lucas, 2000). 
Fig. 2 - Situation géographique du Flageolet I Fig. 2 - Geographic location of Le Flageolet I

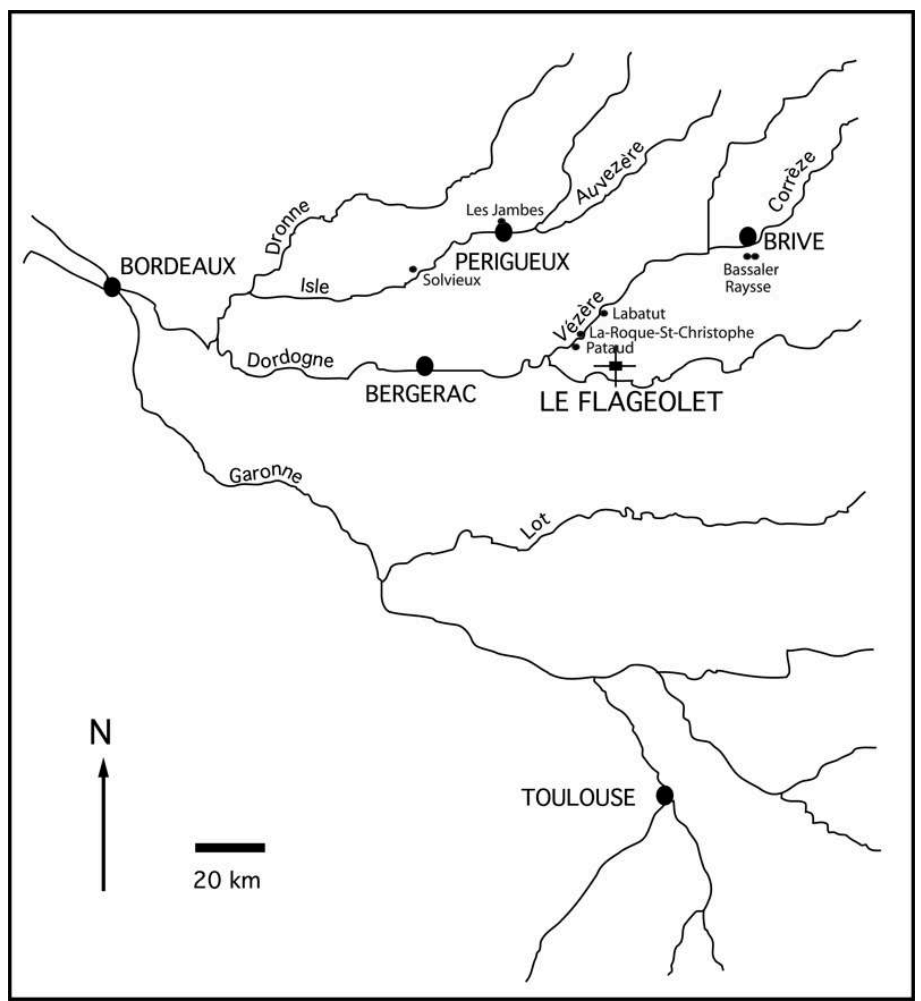

Fig. 3 - Coupe stratigraphique sagittale du Flageolet I, levé : Rigaud (extrait de Rigaud, 1982). Les niveaux XI à VIII sont aurignaciens et les niveaux VII et au-dessus sont gravettiens Fig. 3 - Sagittal section of Le Flageolet I stratigraphy (extracted from Rigaud, 1982). Levels XI to VIII are aurignacian and levels VII and above are gravettian

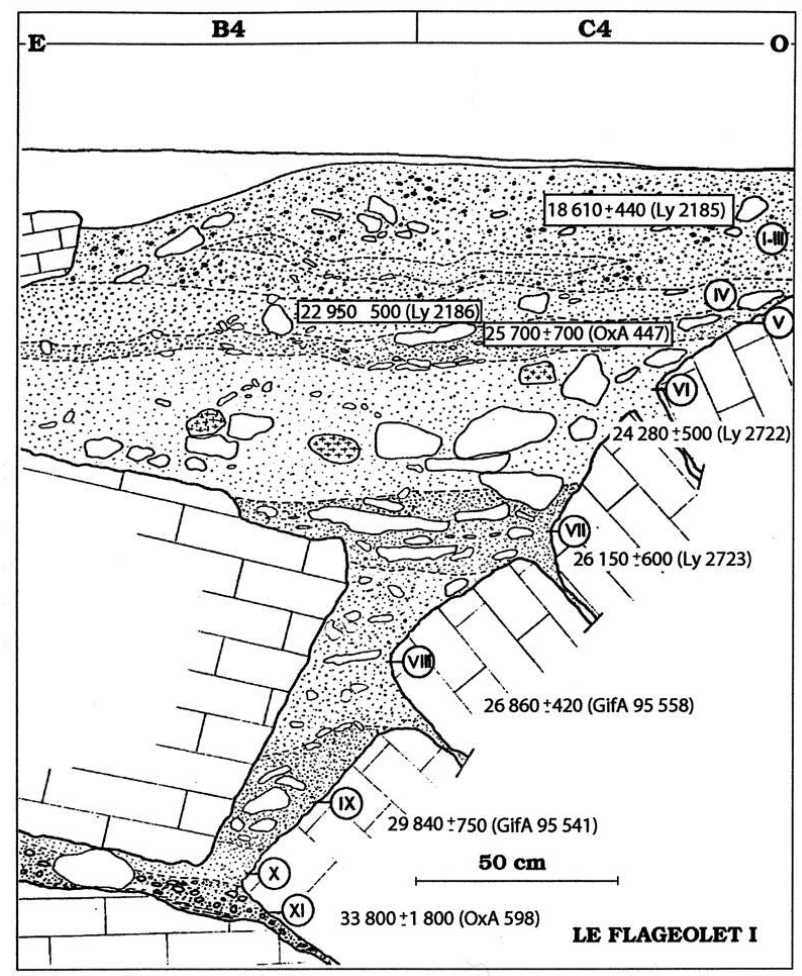




\section{Premières descriptions et interprétations fonctionnelles}

4 La première évocation du burin plan date de 1903 (Bardon et al., 1903). Dès 1911, M. Bourlon l'intègre dans sa première classification des burins (Bourlon, 1911). Ce n'est que plus tard que ce burin va être reconnu en tant que type particulier par L. Pradel qui le signale à la grotte du Raysse (Pradel, 1953). Il le qualifie de burin d'angle et plan dont il détaille les deux stades de fabrication :

1. réalisation d'un burin d'angle et plan et

2. " découronnement de ce burin (comme ferait un avivage), par un enlèvement partant de l'intersection angle et plan pour se diriger obliquement, un peu vers le bas, sur la face opposée aux enlèvements plans, en mordant un peu plus sur les enlèvements d'angle que sur les enlèvements plans » (Pradel, 1966b, p. 48).

$5 \quad$ Les burins du Raysse sont également décrits sous le nom de burin de Bassaler par D. de Sonneville-Bordes considérant que ces outils, nombreux à la Roque-Saint-Christophe, à l'abri Labatut, à l'abri Laraux, caractérisent le Périgordien supérieur à burins de Noailles (Sonneville-Bordes, 1958) et que J. Couchard et elle-même ont pu mettre en évidence à la grotte de Bassaler (Couchard et Sonneville-Bordes, 1960).

Reprenant la définition du burin du Raysse, H.-L. Movius et N.-C. David (1970) décrivent en fait le mode de fabrication qui s'avère plus complexe que ne l'avait décrit L. Pradel. Il peut se résumer comme suit :

- aménagement d'une troncature retouchée

- premier coup de burin

- enlèvement du biseau par une petite retouche tertiaire

- second coup de burin incliné sur la face ventrale

- seconde retouche tertiaire

- troisième coup de burin encore plus incliné sur la face ventrale (Movius et David, 1970).

7 Ils interprètent ce « procédé de fabrication » comme " un témoignage, non pas d'une création d'un type de burin spécialisé et fonctionnellement différent mais, seulement d'une technique de ravivage permettant de tirer l'usage maximum d'un morceau de silex" (Movius et David, 1970, p. 454). Toujours selon ces auteurs, le burin du Raysse est un burin sur troncature retouchée qui a subi une série de ravivages en vue d'obtenir un biseau plus étroit, plus efficace.

\section{Nouvelle interprétation fonctionnelle}

8 En dépit de ce qu'a pu écrire J. Sackett à propos des burins du Raysse de Solvieux (Sackett, 1999, p 248 : « ...even assuming -as seems reasonnable- that the burin was itself an active tool rather than a nucleus for obtaining a particular form of delicate spall which in turn served some special function.»), nous soutenons, comme L. Klaric, l'hypothèse selon laquelle ces burins sont des nucléus à lamelles et que la méthode de ravivage décrite par H.-L. Movius et N.-C. David représente en fait les phases successives d'un débitage lamellaire, la modification tertiaire tenant le rôle de préparation ou de réaménagement du plan de frappe. 
D'autres exemples de débitage lamellaire à partir d'un «burin » peuvent être cités (Buisson, 1991, Bosselin et Djindjian, 1994, Zilhao et al., 1997, Aubry et al., 1997, Lucas, 1997). Au Blot (Cerzat, Haute-Loire), D. Buisson évoque la possibilité de burins périgordiens jouant le rôle de nucléus à lamelles (« Une analyse détaillée des techniques de fabrication des pièces à dos a permis de mettre en évidence l'utilisation fréquente des chutes de burin pour leur confection. Cette observation pose le problème de savoir si les burins du Blot n'ont pas joué le rôle de nucléus à lamelles dans un contexte où l'économie de la matière première était, semble-t-il de bonne règle.» (Buisson, 1991, p. 107)); son travail étant préliminaire, il ne fournit pas de précision sur le type de burin concerné. Ce sont ces burins qui ont fait, quelques années plus tard, l'objet de l'étude déjà citée de Laurent Klaric.

10 A l'abri Pataud (Les Eyzies- de-Tayac), la couche 4inf qui contient des burins de Noailles est sous-jacente à la couche 4 moy, riche à la fois en burins de Noailles et en burins du Raysse, elle-même sous-jacente à la couche 4 sup où seuls les burins du Raysse subsistent. A la suite de H.-L. Movius et N.-C. David (1970) , B. Bosselin et F. Djindjian tentent d'expliquer ce remplacement par une évolution technologique visant le même résultat : la production en série de lamelles (Bosselin et Djindjian, 1994); mais aucune analyse technologique n'a été effectuée pour en éclaircir le déroulement.

11 A Buraca Grande (Portugal), T. Aubry et ses collaborateurs (Aubry et al., 1997) décrivent un débitage sur burin à troncature dont les produits sont très caractéristiques. La face supérieure de ces chutes de burin montre, par l'orientation de ses négatifs antérieurs, un débitage unipolaire. En outre, leur profil est rectiligne ou tend à posséder une convexité vers la face supérieure. L'association de ces deux caractères, débitage unipolaire et profil rectiligne, n'est pas concevable sur des nucléus unipolaires plus " classiques » (Zilhao et al., 1997, Aubry et al., 1997). Remarquons que le nucléus de cette chaîne opératoire est un burin d'angle sur troncature tandis que les burins du Raysse sont des burins d'angle et plans sur troncature. L'orientation différente de la surface de débitage explique, nous allons le voir, pourquoi les chutes de burin trouvées au Flageolet I sont moins rectilignes que celles décrites à Buraca Grande.

12 Au Flageolet I, en l'absence provisoire de remontage de chutes de burin sur leur burin, nous avons recherché des arguments permettant le raccord virtuel probable entre chutes de burin et burins du Raysse. Ces arguments peuvent être d'ordre lithologique (comparaison de la matière première utilisée), d'ordre métrique (comparaison des modules des négatifs d'enlèvements sur les burins avec les dimensions des chutes de burin), d'ordre morphologique (latéralisation des burins et de leurs chutes, torsion des chutes de burin) et d'ordre technologique (préparation du bord latéral du burin avant l'enlèvement des premières chutes, présence de la troncature, préparation du plan de frappe avant le coup de burin).

13 Par la suite, nous nous sommes efforcée de montrer que ces lamelles ont été produites pour être retouchées et pour cela, nous avons confronté la population de lamelles brutes à celles des lamelles retouchées.

14 Nous n'avons cependant pas écarté, dans notre étude, la possibilité d'une utilisation du «burin» en tant qu'outil. 


\section{Analyse}

L'objectif de cette analyse est donc triple :

- tester l'hypothèse selon laquelle les burins du Raysse seraient des nucléus à lamelles,

- confirmer qu'une importante population de lamelles caractéristiques a été produite par ces "burins",

- prouver qu'une partie de cette population a été sélectionnée pour la fabrication d'armatures.

\section{Les burins du Raysse, des nucléus à lamelles}

\section{Choix du support}

Le nombre total de burins du Raysse dans la couche $\mathrm{V}$ du Flageolet I est de 45 (dont 9 burins multiples mixtes). Le support choisi préférentiellement (54\%) est allongé, relativement rectiligne, une lame non-corticale de préférence (Tabl. 1), de façon à avoir des bords latéraux suffisamment réguliers pour être exploités. La largeur et l'épaisseur du support ne semblent pas déterminants dans le choix de ce dernier car une forte variation a été observée (fig. 4).

Fig. 4 - Dimensions des supports utilisés pour les burins du Raysse Fig. 4 - Dimensions of the blanks used for the Raysse burins
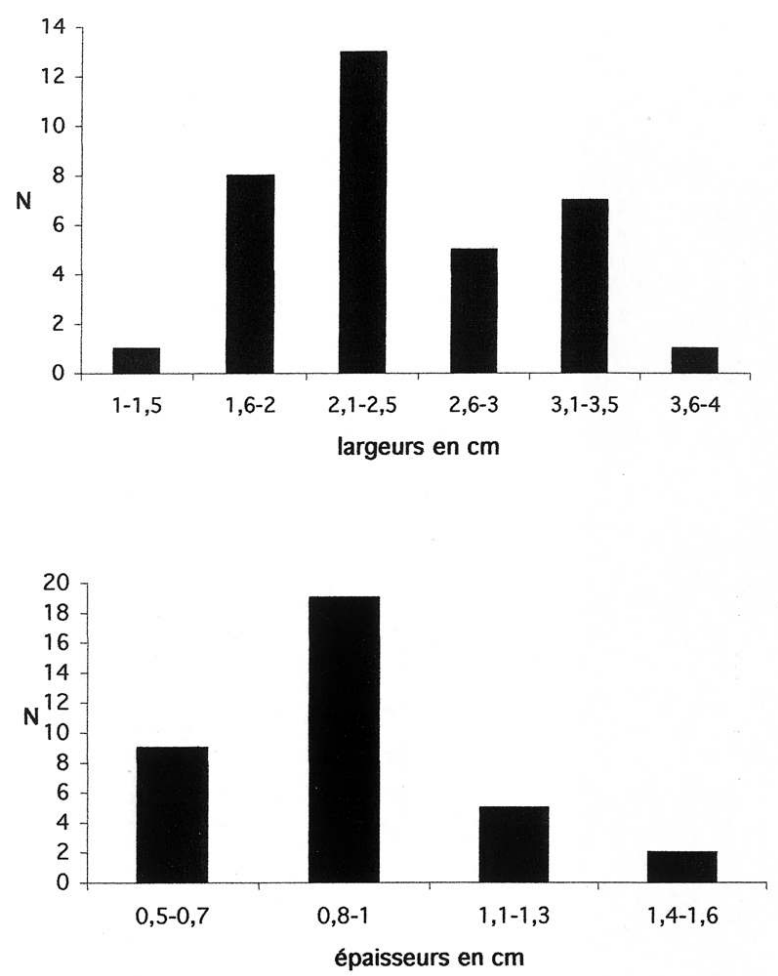

Certains de ces burins sont fabriqués dans un silex patiné très particulier, appelé «silex blanc porcelainé» (Rigaud, 1982) qui serait d'après A. Morala un silex tertiaire (communication personnelle). Ce silex, dont l'origine reste hypothétique, est exclusivement choisi pour ce type de burin dans les couches V et IV. Il est 
pratiquement inexistant dans les autres couches. En effet, avec un taux de $33 \%$, il constitue un des rares exemples de matière première rivalisant avec le silex du Sénonien au Flageolet I (Tabl. 2).

Tabl. 1 - Supports utilisés pour les burins du Raysse $(\mathrm{N}=45)$

Table 1 - Blanks used for the Raysse burins $(\mathrm{N}=45)$

\begin{tabular}{|l|l|}
\hline support & $\%$ \\
\hline lame non-corticale & 54 \\
\hline lame corticale & 7 \\
\hline éclats plus ou moins corticaux & 10 \\
\hline produit de ravivage & 9 \\
\hline indéterminé & 20 \\
\hline
\end{tabular}

Tabl. 2 - Matières premières utilisées pour les burins du Raysse $(\mathrm{N}=45)$

Table 2 - Raw material used for the Raysse burins $(N=45)$

\begin{tabular}{|l|l|}
\hline matière première & $\%$ \\
\hline \hline silex du Sénonien & 51 \\
\hline silex blanc porcelainé & 33 \\
\hline silex du Bergeracois & 9 \\
\hline silex patiné & 7 \\
\hline
\end{tabular}

\section{Mise en place du plan de frappe}

Dans tous les cas observés le plan de frappe est une troncature semi-abrupte; les avantages sont nombreux :

1. elle permet d'aménager l'angulation adéquate entre le plan de frappe et la surface de débitage $\left(<90^{\circ}\right)$,

2. elle favorise la saillie de la zone d'impact indispensable pour la précision du coup de burin

3. elle renforce la solidité du plan de frappe (Klaric, 1999).

19 La direction de la retouche de la troncature, en relation directe avec le type de surface de débitage utilisé, est directe dans la quasi-totalité des cas ( $89 \%, 2 \%$ croisée et $2 \%$ indéterminable); en effet, la retouche de la troncature serait inverse si c'était la face supérieure qui était exploitée ceci afin de permettre une angulation optimale entre le plan de frappe et la surface à débiter (Klaric, 1999). En ce qui concerne l'inclinaison de la retouche, celle-ci semble liée à la morphologie même du support choisi comme nucléus : plus le support sera mince plus la retouche sera abrupte et inversement plus 
le support sera épais plus elle sera développée et ceci afin de maintenir en permanence l'angulation adéquate entre le plan de frappe et la surface de débitage.

A l'état d'abandon, nous avons relevé deux délinéations possibles pour la troncature : rectiligne ou convexe en accent circonflexe; elles correspondent à deux stades successifs dans le processus de débitage des lamelles où le point d'impact du percuteur se déplace de gauche à droite (objet vu de la face supérieure) (fig. $1 \mathrm{n}^{\circ}$ 5). La forme de la troncature va donc traduire l'état d'avancement de la séquence de production sachant qu'une troncature rectiligne correspond au stade 1 et une troncature en forme d'accent circonflexe au stade 2 . Au cours du débitage, les enlèvements qui, au début, étaient d'angle deviennent de plus en plus plans; pour ces derniers, le tailleur a besoin de préparer à nouveau le plan de frappe en dégageant une proéminence sur la troncature qui déterminera le point d'impact (cette modalité correspond à la « modification tertiaire » décrite par H.-L. Movius et N.-C. David formant comme une sorte de museau (Demars, 1973)). En plus d'obtenir un point d'impact plus saillant, la modification tertiaire permet également d'aligner la zone de percussion avec l'axe de débitage voulu (Klaric, 1999). La majorité des burins du Raysse présente une troncature de stade 2 (fig. 1) ; ceci confirme que ce stade représente un état avancé de l'exploitation lamellaire de ce type de nucléus.

\section{Mise en forme du nucléus}

21 Sur une lame-support, les possibilités pour initier le débitage sont assez limitées : le tailleur utilise une nervure de la face supérieure ou le bord, retouché ou non, pour faire « filer » la première lame (Klaric, 1999). Seule la seconde possibilité est décrite pour la couche V du Flageolet I. En effet, l'observation d'une petite retouche de préparation sur le bord latéral gauche des burins est possible mais sur seulement quatre d'entre eux car dans la plupart des cas, cette retouche a été emportée par le premier enlèvement de chute de burin. Elle a donc été recherchée et observée sur les premières chutes de burin débitées. Ces dernières portant cette retouche de préparation qui facilite leur détachement peuvent être assimilées aux lames à crête premières du débitage laminaire. Elles semblent surtout corriger la "déviation latérale de la carène " (appelée également « flèche ») liée à la courbure du support (Klaric, op. cit.).

\section{Débitage des lamelles}

Dans son étude des burins du Blot, Laurent Klaric a choisi d'élargir la définition du terme de «débitage plan» initialement introduit par D. de Sonneville-Bordes et J. Perrot (1956) pour désigner un enlèvement oblique ou presque parallèle détaché de la face d'éclatement du support servant de nucléus. Il observe suivant les cas une exploitation simultanée des faces supérieure et inférieure du support (Klaric, 1999).

$\mathrm{Au}$ Flageolet I, la surface de débitage exploitée est la face inférieure; pour diverses raisons, le tailleur n'a pas jugé nécessaire d'étendre l'exploitation du nucléus à toutes les surfaces offertes par le support (la morphologie des lamelles obtenues sur la face supérieure ou sur la face inférieure n'est certainement pas identique et ne permet sans doute pas la même utilisation).

En revanche, le débitage plan sur la face inférieure est toujours associé au débitage d'angle qui permet de commencer la production des lamelles en utilisant l'angle du 
bord de la lame-support formé par la face d'éclatement et la face supérieure. Nous observons donc sur les burins du Raysse du Flageolet I une rotation progressive de la surface de débitage depuis l'angle formé par le bord du support vers la face inférieure.

Les contraintes rencontrées lors de ce type de débitage sont principalement dues à la morphologie de la face inférieure de la lame qui est lisse, plutôt concave (absence de cintre et de carène) mais également parfois convexe (présence du bulbe). Le tailleur va donc, pour guider la lamelle qu'il s'apprête à détacher, utiliser l'arête la plus éloignée du bord de la lame laissée par l'enlèvement précédent. Notons que la rotation progressive de la surface de débitage vers la face inférieure, plane, rend tout de même de plus en plus difficile le détachement des lamelles (Klaric, op. cit.) ce qui explique le petit nombre d'enlèvements lamellaires observables sur chacun des burins (5 à 6). Laurent Klaric remarque également que la concavité de la face d'éclatement empêche l'obtention de longues lamelles tandis que celles obtenues sur la face supérieure ne subissant pas cette contrainte sont en général plus allongées; ceci confirme que, suivant les faces exploitées de la lame-support, les lamelles ont des morphologies différentes.

Il faut également remarquer que le plan de frappe (c'est-à-dire la troncature) est très nettement latéralisé : nous constatons que $91 \%$ des burins présentent des enlèvements à gauche opposés à la troncature qui est à droite (objet vu de la face supérieure) (8\% à droite et $1 \%$ indéterminés). Un tailleur droitier sera plus à l'aise pour débiter les lamelles sur la face inférieure en tenant la lame-support du nucléus, la face inférieure vers lui et en appliquant le coup de percuteur à l'intersection de la troncature et du bord latéral gauche (bord droit si on considère la lame au moment du débitage).

\section{Abandon des nucléus}

La moitié des pièces observées est cassée; parmi ces fragments de burin, la plupart présente un dernier enlèvement réfléchi. Il nous paraît possible que cette cassure ait pu être voulue par le tailleur qui aurait pu ainsi remettre en forme un plan de frappe par une troncature sur la surface de cassure et reprendre le débitage d'une autre série de lamelles. Cette hypothèse est confirmée par la faible dimension des burins du Raysse entiers présentant un accident de débitage (en général un réfléchissement) : trop courts pour être cassés et remis en forme, ils ont été soit abandonnés tels quels soit exploités sur l'autre extrémité du support lorsque cela était possible (la présence d'un bulbe ou d'ondulations sur la face inférieure rendent la surface de débitage souvent trop convexe ou irrégulière pour être exploitée). A côté de ces burins cassés volontairement, nous avons également observé des fragments de burins ne présentant aucun accident de débitage; ces fragments sont en général plus courts et nous leur attribuons plus naturellement une cause accidentelle.

Il semble à présent très probable que les burins du Raysse soient des nucléus ; il nous a donc paru indispensable, afin d'avoir une vision plus juste de leur place dans l'équipement technique des préhistoriques, de soumettre ces objets à une analyse tracéologique. Celle-ci fut confiée à Maureen A. Hays qui, en raison de la surface très patinée, n'a malheureusement pu étudier que dix pièces sur 45 . Les résultats de ces observations ne sont donc qu'indicatifs en attendant que des analyses soient appliquées à un échantillon plus important prélevé dans une série moins patinée. Sur ces dix objets, sept ne portent aucun micropoli, un est trop patiné pour être observable, un 
présente sur le biseau des traces associées à la production des lamelles (abrasion du plan de frappe) et le dernier présente des traces d'utilisation indéterminées localisées sur le bord de la lame-support (Hays, communication personnelle).

\section{Les chutes de burin produites par les burins du Raysse}

Les 1156 chutes de burin de la couche V, prélevées sur la totalité du matériel (refus de tamis inclus), ont été triées en partant du principe que les burins étudiés étant latéralisés à gauche, les chutes de burin devaient l'être également. Nous avons donc séparé de la population de chutes de burin totale les chutes de burin "latéralisées à gauche " mais pour latéraliser une chute de burin, il faut l'orienter; or, l'état de fragmentation et les faibles dimensions de ces objets rendent l'observation difficile et nous avons dû établir un certain nombre de critères diagnostiques.

Avant d'orienter latéralement une chute de burin (c'est-à-dire de déterminer si elle a été débitée à droite ou à gauche) il est nécessaire de l'orienter verticalement (c'est-àdire de distinguer le haut du bas par rapport au sens de débitage). Cette diagnose est donc composée de deux étapes qui sont indissociables (figure 5 où les chutes de burin ont volontairement été orientées suivant le sens de débitage) : en effet, il est tout d'abord nécessaire d'observer sur le fragment étudié au moins un des critères servant à l'orientation verticale de la chute de burin puis un autre des critères définis pour l'orientation latérale. Prenons par exemple

1. la présence du bulbe de percussion caractéristique d'un fragment proximal ;

2. la présence de la portion de la face inférieure du support observable sur une face supérieure de chute de burin.

31 Remarquons que le bord de la lame-support est souvent retouchée : c'est un angle aigü qui ne peut pas être confondu avec celui, plus obtus, des négatifs d'enlèvements de chutes de burin détachées antérieurement. Pour préciser le sens de débitage, toujours unipolaire, les ondulations et les lancettes des négatifs antérieurs de chutes de burin sont également à considérer. Nous avons remarqué que les fragments mésiaux sont très difficiles à latéraliser car les lancettes et les ondulations y sont moins visibles que sur les fragments distaux. 
Fig. 5 - Orientations verticale et latérale d'une chute de burin Fig. 5 - Vertical and lateral orientations of a burin spall

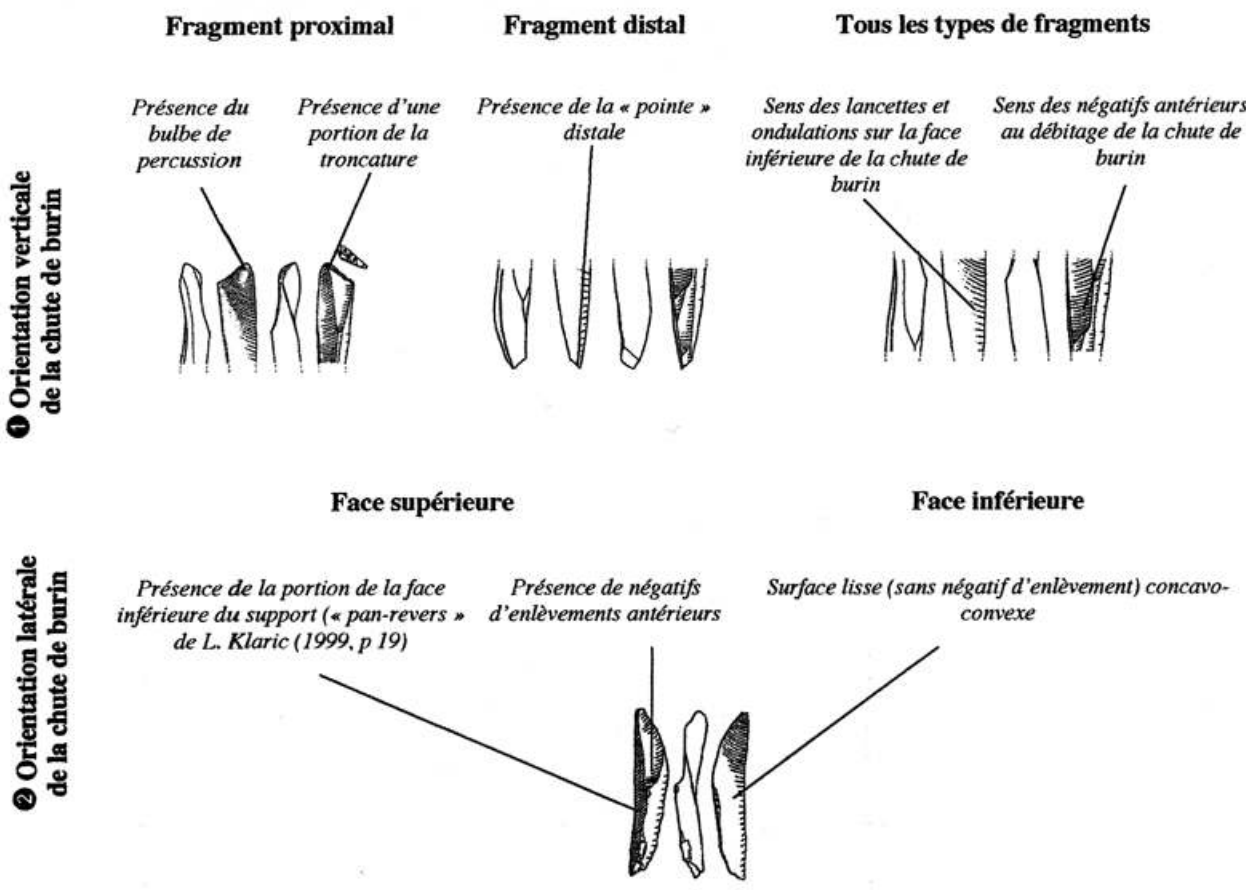

(d'après des dessins de J.-G. Marcillaud)

(after drawings by J.-G. Marcillaud)

Les résultats du tri sont les suivants :

- 250 chutes de burin latéralisées à droite (21\%),

- 557 à gauche (49\%),

- 349 sont indéterminables (30\%).

La population totale de 1156 chutes de burin regroupe donc, en plus des lamelles issues des burins du Raysse, des lamelles venant d'autres types de burins, très nombreux dans ce niveau.

34 Tous les burins du Raysse étant latéralisés à gauche (voir supra : débitage des lamelles), la population de lamelles étudiée sera donc constituée des 557 chutes de burin latéralisées à gauche elles aussi.

\section{Filiation technologique de ces lamelles avec les burins du Raysse}

Différents critères d'ordre morphométrique, technologique ou lithologique permettent de confirmer que les burins du Raysse sont à l'origine de la production de la grande quantité de lamelles recueillies dans la couche V du Flageolet I.

\section{Dimensions}

Les burins du Raysse ne sont pas les seuls burins présents dans l'ensemble lithique étudié : c'est pourquoi, afin de confirmer que seuls ces «burins" très particuliers sont à l'origine d'une production systématique de lamelles, il est nécessaire de mener une 
étude comparative des dimensions des derniers négatifs d'enlèvement des burins du Raysse et des burins d'axe et d'angle sur troncature retouchée. Nous avons choisi ces deux types de burins pour deux raisons :

1. ils sont, avec les burins du Raysse, relativement nombreux dans la couche étudiée (pour les burins d'axe sur troncature retouchée, $\mathrm{n}=48$ et les burins d'angle sur troncature retouchée, $\mathrm{n}=38$ );

2. étant, comme les burins du Raysse, des burins sur troncature, ils ont produit des chutes de burin également susceptibles de présenter une portion de cette troncature au niveau de leur talon.

37 Les négatifs d'enlèvements lamellaires lus sur les biseaux des burins d'axe ou d'angle sur troncature retouchée apparaissent plus larges (largeur maximale 1,6 cm) et plus longs (longueur maximale $7 \mathrm{~cm}$ ) que les négatifs lus sur les burins du Raysse (largeur maximale $1,1 \mathrm{~cm}$ et longueur maximale $3,5 \mathrm{~cm}$ ) (fig. 6). Ceci constitue un premier argument pour penser que seul un de ces deux types de burins est à l'origine de la production lamellaire étudiée ici. Les résultats montrent que les négatifs issus des burins sur troncature « classiques » (burins d'axe et d'angle sur troncature retouchée) s'avèrent être plus longs et plus larges $(2,36 \mathrm{~cm}$ de longueur moyenne et $0,64 \mathrm{~cm}$ de largeur moyenne) que ceux des burins du Raysse (2,08 cm de longueur moyenne et 0,59 $\mathrm{cm}$ de largeur moyenne) (fig. 6). En outre, les négatifs des burins d'axe et d'angle sur troncature retouchée ne présentent pas la régularité et l'homogénéité dimensionnelle caractéristiques d'une production de supports en série ; ceci est certainement lié au fait que les supports de ces burins n'ont pas fait l'objet d'une sélection rigoureuse (utilisation de lames et d'éclats corticaux, ou non, aux dimensions et morphologies diverses). De plus, les burins d'axe et d'angle sur troncature ne présentent pas de troncature franchement latéralisée ( $57 \%$ à droite et $43 \%$ à gauche) contrairement aux burins du Raysse ( $91 \%$ de cas où la troncature est à droite, $8 \%$ à gauche et $1 \%$ indéterminée). Nous pensons donc que les burins du Raysse sont probablement à l'origine de la production lamellaire décrite ici. Les négatifs d'enlèvements lamellaires lus sur les biseaux des burins du Raysse présentent en effet des valeurs minimales réduites ( $1 \mathrm{~cm}$ de long et 0,3 de large (fig. 6)) ; leur longueur moyenne est de 2,08 cm et leur largeur moyenne de $0,59 \mathrm{~cm}$. La longueur moyenne des lamelles est de $2,89 \mathrm{~cm}$ et leur largeur moyenne de $0,59 \mathrm{~cm}$. La comparaison des deux nuages de points (fig. 7) permet de constater que la répartition des modules des négatifs d'enlèvements de chutes de burin correspond à celle des modules des chutes de burin non-retouchées entières. Les moyennes des négatifs sont plus faibles car ce sont les derniers négatifs produits sur la surface de débitage. En effet, les premières chutes de burin sont souvent plus longues parce que d'une part, le burin est plus long et d'autre part, elles filent mieux et plus loin le long du bord préparé. 
Fig. 6 - Modules longueur/largeur des derniers négatifs d'enlèvements lamellaires sur les burins d'axe et d'angle sur troncature retouchée et sur les burins du Raysse, couche V, Flageolet I Fig. 6 - Length/width modules of the last bladelet negative removal of burins on axis, on angle on retouched truncation and of Raysse burins, level V, Flageolet I

\section{largeurs en $\mathrm{cm}$}

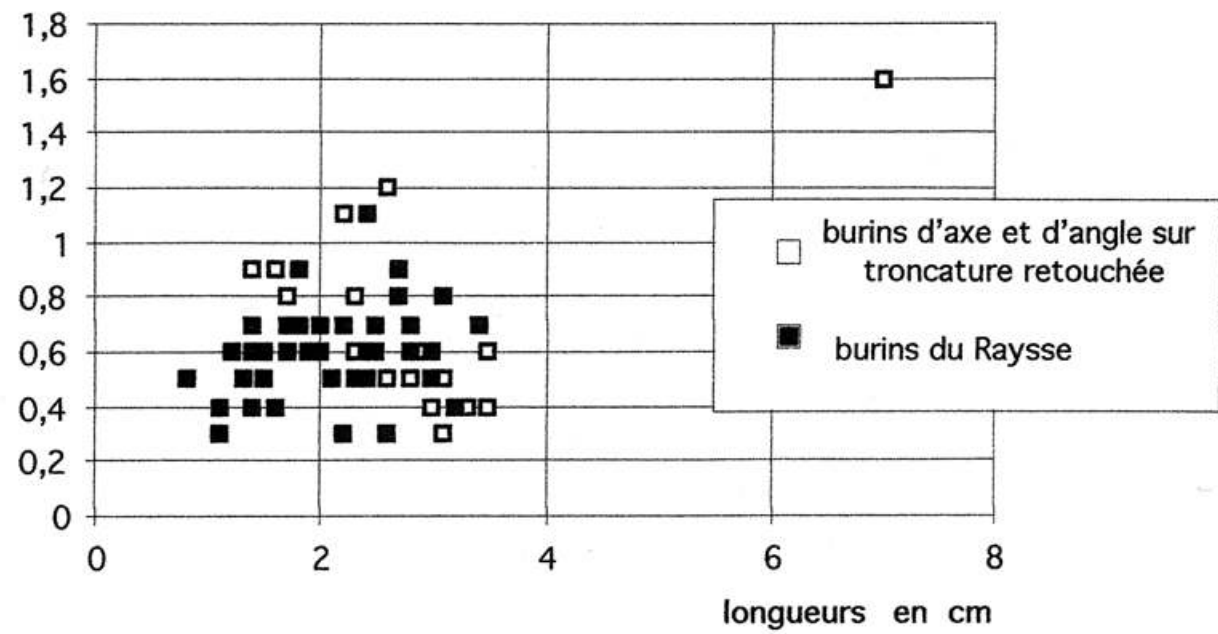

Fig. 7 - Modules longueur/largeur des chutes de burin latéralisées à gauche et des derniers négatifs d'enlèvements lamellaires sur les burins du Raysse, couche V, Flageolet I

Fig. 7 - Length/width modules of the left-sided burin spalls and of the last bladelet negative removal of Raysse burins, level V, Flageolet I

\section{largeurs en $\mathrm{cm}$}

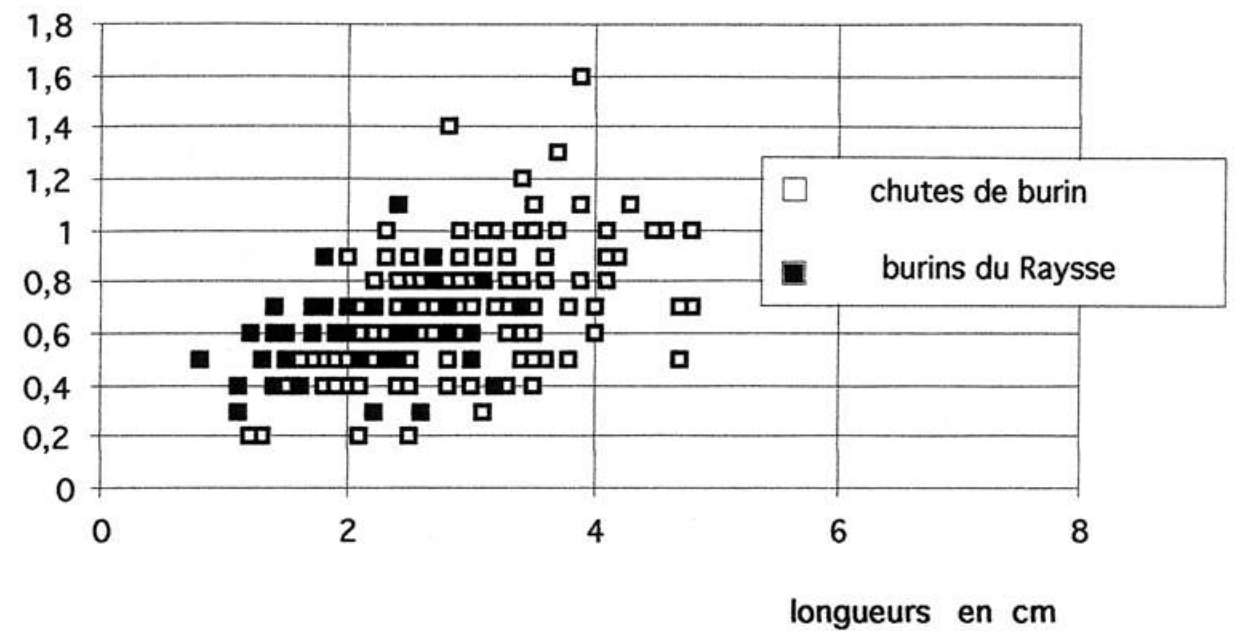

(extrait de Lucas, 2000)

(extracted from Lucas, 2000) 


\section{Matière première}

Parmi cette population de chutes de burin latéralisées à gauche, 27 \% d'entre elles sont en silex blanc porcelainé ; ce taux est proche de celui des burin du Raysse taillés dans ce même matériau (33\%) par rapport à l'ensemble de l'outillage (Tabl.3). Si l'on ne considère que la population de chutes de burin latéralisées à droite, seulement $3 \%$ d'entre elles sont en silex blanc porcelainé ce qui confirme également, mais indirectement, l'étroite relation entre cette matière première et les burins du Raysse.

Tabl. 3 - Matières premières utilisées pour les chutes de burins latéralisées à gauche $(\mathrm{N}=557)$

Table 3 - Raw material used for the left-sided burin spalls $(N=557)$

\begin{tabular}{|l|l|}
\hline matière première & $\%$ \\
\hline silex du Sénonien & 57 \\
\hline silex blanc porcelainé & 27 \\
\hline silex du Bergeracois & 2 \\
\hline \hline indéterminé & 14 \\
\hline
\end{tabular}

\section{Morphologie du talon}

Un certain nombre de ces chutes de burin présente, au niveau du talon, une portion de la troncature du burin qui a été enlevée lors du coup de burin ( $40 \%$ des lamelles entières et des fragments proximaux). La quasi-totalité des lamelles ( $90 \%$ ) sur lesquelles subsiste un reste de troncature est torse. Cette torsion, différente de celle présentée par les lamelles Dufour, est induite par la troncature qui forme un angle avec le bord latéral gauche de la chute de burin (fig. $8 \mathrm{n}^{\circ} 3$ ). Le bord gauche de la lamelle se relève vers la face supérieure au niveau de cet angle ${ }^{2}$. Parmi ces chutes de burin présentant une portion de troncature, $43 \%$ sont en silex blanc porcelainé. Ce taux, plus fort que celui des chutes de burin simplement latéralisées à gauche (27\%), montre que la présence de la troncature caractérise bien les chutes issues des burins du Raysse. Toutes les chutes de burin ne sont pas torses car seules celles qui sont entières ou proximales ont pu conserver la portion de troncature. C'est pourquoi les fragments mésiaux ou distaux sont rarement torses. 
Fig. 8 - Chutes de burins du Raysse, couche V, Flageolet I

Fig. 8 - Raysse burin spalls, level V, Flageolet I

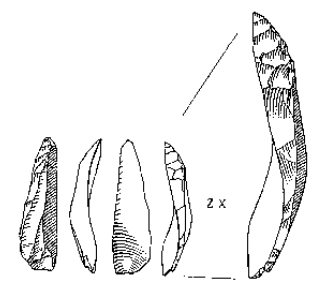

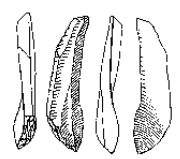

सित्य

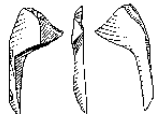

$1 \mathrm{~cm}$
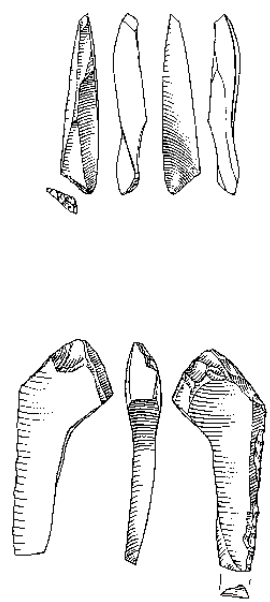

La pièce $n^{\circ} 1$ montre une préparation de la lame-support avant le détachement de la chute de burin. Les pièces $n^{\circ} 2$ et 3 présentent sur leur talon une portion de la troncature du burin induisant la torsion générale du support. Les pièces 4 et 5 sont les parties distales de chutes outrepassées de burins du Raysse doubles

Object $n^{\circ} 7$ illustrates preparation of the blade edge before the removal of the burin spall. Objects $n^{\circ} 2$ and 3 have, on their butts, a segment of the burin truncation implying the general torsion of the blank. Objects $n^{\circ} 4$ and 5 illustrate the distal portion of plunging burins spalls from double Raysse burins

(dessins de J.-G. Marcillaud).

(drawings by J.-G. Marcillaud). 
Fig. 9 - Chutes de burins du Raysse retouchées, couche V, Flageolet I : $\mathrm{n}^{\circ} 1$ à 7 dessins de S. Pasty et $\mathrm{n}^{\circ} 8$ à 10 dessins de J.-G. Marcillaud

Fig. 9 - Retouched Raysse burin spalls, level V, Flageolet I: $n^{\circ} 1$ to 7 drawing by S. Pasty and $n^{\circ} 8$ to 10 drawings by J.-G. Marcillaud

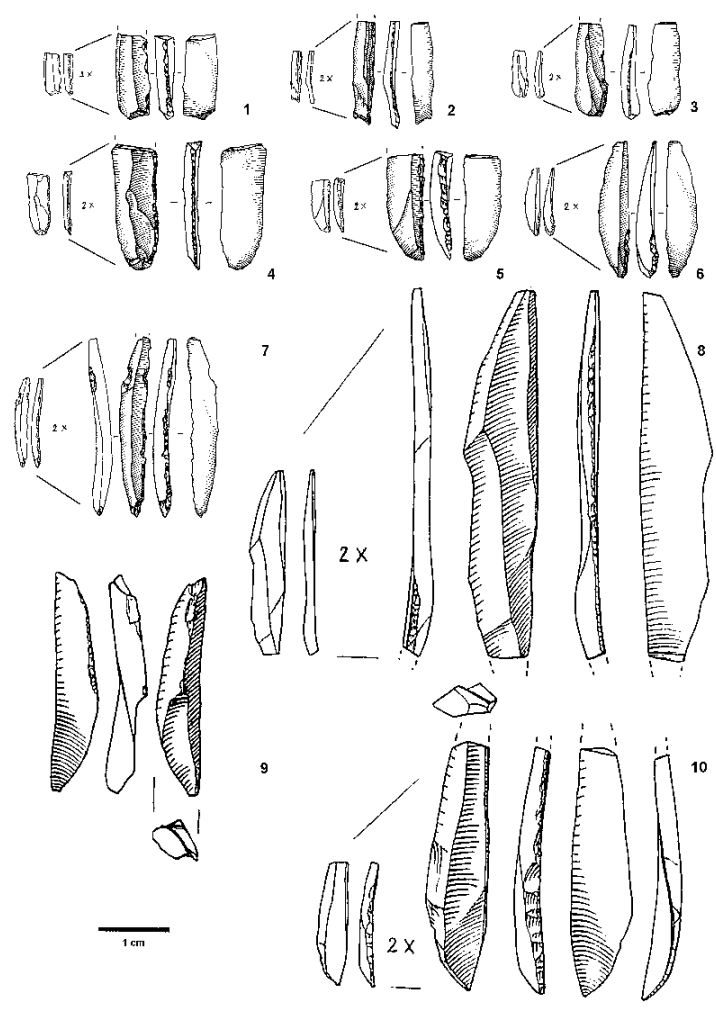

\section{La préparation du bord latéral}

Quant à la retouche de préparation sur le bord latéral gauche, $33 \%$ des lamelles en présentent des traces : il s'agit des premières lamelles débitées, les suivantes ont suivi les nervures-guides des enlèvements précédents.

\section{Sélection et retouche de ces lamelles}

Les chutes de burin retouchées ont toutes été inventoriées sous le nom de lamelles à fine retouche directe ou inverse sur un bord (L. Klaric a proposé de les appeler lamelles de La Picardie (Klaric et al., 2001)). Ces dernières, qui présentent une retouche directe ou inverse, sont au nombre de 46 (fig. 9). Mais toutes les lamelles à fine retouche directe ou inverse sur un bord ne sont pas nécessairement des chutes de burin retouchées (Tabl.4). D’après les critères évoqués précédemment pour déterminer ces supports, seulement $60 \%$ d'entre elles le sont.

Un certain nombre d'arguments permet d'affirmer que les lamelles produites par les burins du Raysse ont été sélectionnées pour être retouchées et probablement utilisées.

Tabl. 4 - Supports utilisés pour les lamelles finement retouchées $(N=46)$

Table 4 - Blanks used for the finely retouched bladelets $(\mathrm{N}=46)$

\begin{tabular}{|l|l|}
\hline support & $\%$ \\
\hline
\end{tabular}




\begin{tabular}{|l|l|}
\hline chute de burin & 60 \\
\hline lamelle classique & 34 \\
\hline indéterminé & 6 \\
\hline
\end{tabular}

\section{Dimensions}

43 Seules quatre d'entre elles sont entières; elles ont une longueur moyenne de 2,2 cm. La largeur moyenne de ces lamelles retouchées est de 0,53 cm. Ces dimensions se situent dans le domaine de variation des négatifs d'enlèvements des burins et des chutes de burin non-retouchées (fig. 6 et 7).

\section{Latéralisation}

La totalité de ces chutes de burin est latéralisée à gauche.

\section{Matière première}

Le taux de silex blanc porcelainé est de $25 \%$ (Tabl. 5).

Tabl. 5 - Matières premières utilisées pour les lamelles finement retouchées $(N=46)$

Table 5 - Raw material used for the finely retouched bladelets $(\mathrm{N}=46)$

\begin{tabular}{|l||c|}
\hline matière première & $\%$ \\
\hline silex du Sénonien & 59 \\
\hline silex blanc porcelainé & 25 \\
\hline indéterminé & 16 \\
\hline
\end{tabular}

\section{Morphologie du talon}

Une portion de troncature est présente sur $50 \%$ des fragments proximaux et des lamelles entières.

\section{Discussion et conclusions}

Il est important de remarquer qu'on ne peut pas effectuer de comparaison directe entre les lamelles finement retouchées et la population de chutes de burin latéralisées à gauche car cette dernière ne regroupe pas seulement des chutes de burin issues des burins du Raysse (les autres burins sur troncature ont également pu produire des chutes de burin latéralisées à gauche sans qu'il s'agisse pour autant d'une production systématique). Il est cependant possible d'évaluer le nombre de chutes de burin issues 
des burins du Raysse en se référant au nombre de lamelles torses (Tabl. 6: 60\% soit environ 330 chutes de burin torses) et de calculer le nombre de chutes de burin minimal (car toutes les lamelles produites ne sont pas forcément torses) produites par biseau qui est d'environ 5 à 6 lamelles ( $\mathrm{n}=330$ chutes de burin/56 biseaux), soit 10 à 12 chutes par burin double (4). Il s'agit d'un nombre minimum évalué uniquement d'après la quantité de lamelles torses; sachant que ces lamelles n'étaient pas exactement les lamelles recherchées, la quantité de lamelles totales était certainement bien plus élevée (5). De plus, étant donnée la longueur moyenne des lames-supports retrouvées $(4,5 \mathrm{~cm})$, ces nucléus à lamelles ont, nous avons vu, très probablement subi des réavivages successifs dont l'importance est difficile à évaluer.

Tabl. 6 - Morphologie des chutes de burin latéralisées à gauche $(\mathrm{N}=557)$ Table 6 - Left-sided burin spall morphology $(\mathrm{N}=557)$

\begin{tabular}{|l||l|}
\hline morphologie & $\%$ \\
\hline torse & 59 \\
\hline plane & 23 \\
\hline courbe & 15 \\
\hline indéterminé & 3 \\
\hline
\end{tabular}

Un certain nombre de lamelles produites par les burins du Raysse ont été sélectionnées pour être retouchées. Si ce fait semble établi, deux problèmes subsistent :

1. seul un petit nombre de lamelles a été retouché (26),

2. des lamelles torses ont été produites mais ne semblent pas avoir été par la suite sélectionnées.

Le petit nombre de chutes de burin retouchées pourrait s'expliquer par une utilisation particulière s'effectuant hors du site, à la suite de laquelle seule une petite fraction d'entre elles sont ramenées, souvent fragmentées (seulement $9 \%$ sont entières), par le biais de processus divers (par exemple enfouies dans la carcasse de l'animal ou enchassées sur un fût dans le cas d'une utilisation comme élément de pointe de projectile). Il s'agit toutefois d'hypothèses qui doivent être vérifiées. Il est également possible que les lamelles aient été utilisées non-retouchées. La retouche des lamelles a pour but souvent de modifier la morphologie (voir les lamelles Dufour aurignaciennes (Bon 2000)) et si la modification n'est pas nécessaire, la retouche est absente; dans ce cas seules les analyses tracéologiques peuvent apporter des éléments de réponse.

Les résultats de l'analyse des lamelles retouchées montrent que ces dernières ne sont pas préférentiellement torses (une portion de troncature est présente sur seulement $50 \%$ des fragments proximaux et des lamelles entières); contrairement aux lamelles Dufour, cette caractéristique ne semble pas avoir été spécialement recherchée. Dans ce cas, cette morphologie particulière n'a probablement joué aucun rôle pour le choix du type d'emmanchement. Le critère de sélection de ces lamelles est peut-être davantage d'ordre dimensionnel que morphologique. En revanche, dans le cas où toutes les 
lamelles utilisées ne sont pas retouchées, il est difficile d'avoir une idée précise de la morphologie des lamelles recherchées.

D'une façon plus générale ces données nouvelles concernant le rôle des burins du Raysse permettent de constater que la production lamellaire au Gravettien présente une grande variabilité. Un certain nombre d'objets considérés jusqu'à présent comme des outils sont en fait des nucléus à lamelles. Au Flageolet I, nous n'avons pu mettre cela en évidence que pour un type de burin bien particulier, mais il semble que dans d'autres gisements (le Blot par exemple (Klaric, 1999)), d'autres types de burin soient également concernés par ces considérations fonctionnelles.

Cela nous conduit à soulever le problème d'équifinalité rencontré fréquemment au cours de l'étude des objets lithiques du Paléolithique: en l'absence d'étude technofonctionnelle, il est difficile d'attribuer une fonction d'outil ou de nucléus à tel ou tel objet (exemple des pièces carénées (Hays et Lucas, 2000a), ou des pièces esquillées (Hays et Lucas, 2000b, Lucas et Hays, 2000)). C'est pourquoi il nous semble essentiel de combiner les études technologiques pour démontrer la fonction éventuelle de nucléus et les analyses tracéologiques pour vérifier la fonction d'outil. Il est également important de remarquer que des résultats positifs dans ces deux domaines mettent en évidence un processus de recyclage intéressant d'un point de vue économique (Hays et Lucas, 2000a).

\section{BIBLIOGRAPHIE}

ALAUX J.-F. 1967 - Burins du type « Le Raysse » de l'abri des Battuts (Tarn). Bulletin de la Société Préhistorique Française, 64, n 8, p. 242-247.

AUBRY T., ZILHAO J., ALMEIDA F. et FONTUGNE M.1997 - Production d'armatures microlithiques pendant le Paléolithique supérieur et le Mésolithique au Portugal. In : R. De Balbìn Berhmann and P. Bueno Ramìrez (Ed.), II, Congreso de Arqueologia peninsular, Paleolitico y Epipaleolitico, p. 259-271, Zamora, 24-27 sept. 1996, t 1.

BARDON L., BOUYSSONIE J. et BOUYSSONIE A.1903 - Un nouveau type de burin. Revue d'Anthropologie, 13, p. 165-168.

BON F. 2000 - La question de l'unité technique et économique de l'Aurignacien : réflexions sur la variabilité des industries lithiques à partir de l'étude comparée de trois sites des Pyrénées françaises: La Tuto de Camalhot, Régismont-le-Haut et Brassempouy. Thèse de Doctorat, Université Paris I.

BOSSELIN B. et DJINDJIAN F. 1994 - La chronologie du Gravettien français. Préhistoire Européenne, 6, p. 1-40.

BOURLON M. 1911 - Essai de classification des burins, leurs modes d'avivage. Revue Anthropologique, 21, p. 267-278.

BUISSON D. 1991 - Le Périgordien du Blot (Cerzat, Haute-Loire), note préliminaire. Bulletin de la Société Préhistorique Française, 88, nº 4, p. 104-108. 
CELERIER G. 1967 - Le gisement périgordien supérieur des Jambes, commune de périgueux (Dordogne). Bulletin de la Société Préhistorique Française, 64, n 1, p. 53-82.

COUCHARD J. et SONNEVILLE-BORDES D. de 1960 - La grotte de Bassaler-nord, près Brive, et la question du Périgordien II en Corrèze. L'Anthropologie, 64, n 5-6, p. 415-437.

DEMARS P.-Y. 1973 - Morphologie des burins sur troncature des niveaux périgordiens Vc de la grotte de pré-Aubert (près de Brive, Corrèze). Bulletin de la Société Préhistorique Française, $70, \mathrm{n}^{\circ} 2$, p. 43-50.

HAYS M.-A. et LUCAS G. 2000a - A technological and functional analysis of carinates. Journal of Field Archaeology, 27, $\mathrm{n}^{\circ}$ 4, p. 1-11.

HAYS M.-A. et LUCAS G. 2000b - Pièces esquillées from Le Flageolet I (Dordogne, France) : tools or cores ?. In: S. MacPherron and Lindly(Ed.) Tools or Cores? The identification and study of alternative core technology in lithic assemblages. Paper presented at the 65 th meeting of the Society of American Archaeology, Philadelphie.

KLARIC L. 1999 - Un schéma de production lamellaire original dans l'industrie gravettienne de l'ensemble moyen du gisement du Blot à Cerzat (Haute-Loire). Mémoire de DEA, Université de Paris I, PanthéonSorbonne.

KLARIC L. 2000 - Notes sur la présence de lames aménagées par technique de Kostienki dans les couches gravettiennes du Blot (Cerzat, Haute-Loire). Bulletin de la Société Préhistorique Française, 97, $\mathrm{n}^{\circ} 4$, p. 625-636.

KLARIC L., AUBRY T. et WALTER B. 2001 - Des burins du Raysse : pour quoi faire ? In Matière première, technologie, tracéologie (session générale de la section 6 : Paléolithique supérieur). XIVème Congrès de l'Union Internationale des Sciences Préhistoriques et Protohistoriques, Liège.

LE MIGNOT Y. 2000 - La question de la production d'armatures sur le site gravettien de PlasennAl-Lomm (Ile de Bréhat, Côtes d'Armor). Revue Archéologique de l'Ouest, 17, p. 7-24.

LUCAS G. 1997 - Les lamelles Dufour du Flageolet I (Bezenac, Dordogne) dans le contexte aurignacien. Paléo, 9, p. 191-220.

LUCAS G. 2000 - Les industries lithiques du Flageolet I (Dordogne) : approche économique, technologique, fonctionnelle et analyse spatiale. Thèse de Doctorat, Université de Bordeaux 1.

LUCAS G. et HAYS M.-A. 2000 - Les pièces esquillées du Flageolet I : outils ou nucléus ? In Approches fonctionnelles en Préhistoire. XXVème Congrès Préhistorique de France, Nanterre.

MELLARS P.-A., BRICKER H.-M., GOWLETT J.-A. et EDGES R.-E.-M. 1987 - Radiocarbon accelerator dating of French upper paleolithic sites. Current Anthropology, 28, n 1, p. 128-133.

MOVIUS H.-L. et DAVID, N.-C. 1970 - Burins avec modification tertiaire du biseau, burins-pointes et burins du Raysse à l'abri Pataud, Les-Eyzies (Dordogne). Bulletin de la Société Préhistorique Française, 67, n 2, p. 445-455.

PRADEL L. 1953 - Précisions sur les burins d'angle et les burins plans. Congrès Préhistorique de France. XIV ème session, Strasbourg-Metz, p. 545-552.

PRADEL L. 1965 - Burins « d'angle et plans » et le type du Raysse. Bulletin de la Société Préhistorique Française, comptes-rendus mensuels, 2, p. 54-58.

PRADEL L. 1966a - Classification des burins avec notation chiffrée. Bulletin de la Société Préhistorique Française, 63, n 3, p. 485-500. 
PRADEL L. 1966b - A propos du burin du Raysse. Bulletin de la Société Préhistorique Française, comptes-rendus mensuels, 2, p. 47-49.

PRADEL L. 1971 - Précisions sur le burin du Raysse. Bulletin de la Société Préhistorique Française, 68, $\mathrm{n}^{\circ}$ 9, p. 266.

PRADEL L. 1977 - Définition des burins de Noailles et du Raysse. Bulletin des Amis du GrandPressigny, 28, p. 9-11.

PRADEL L. et PRADEL J.-H. 1966 - La station paléolithique du Raysse, commune de Brive (Corrèze). L'Anthropologie, 70, n 3-4, p. 225-254.

RIGAUD J.-Ph. 1982 - Le Paléolithique en Périgord : les données du sud-ouest sarladais et leurs implications. Thèse de Doctorat d'Etat, Université de Bordeaux I.

RIGAUD J.-Ph. 1993 - L'Aurignacien dans le sud-ouest de la France : bilan et perspectives. In Aurignacien en Europe et au Proche-Orient, p. 181-186. Actes du XII ème Congrès International des Sciences Préhistoriques et Protohistoriques, Bratislava, 1-7 sept. 1991. Institut Archéologique de l'Académie Slovaque des Sciences.

SACKETT J. 1999 - The archaeology of Solvieux : an upper palaeolithic open air site in France. Monumenta Archaeologia vol. 19. Institute of Archaeology, University of California, Los-Angeles. SONNEVILLE-BORDES D. de 1958 - Problèmes généraux du Paléolithique supérieur dans le sudouest de la France. L'Anthropologie, 62, p. 413-451.

SONNEVILLE-BORDES D. de et PERROT J. - 1956. Lexique typologique du Paléolithique supérieur. Outillage lithique IV : Burins. Bulletin de la Société Préhistorique Française, 53, n 7-8, p. 408-412.

ZILHÃO J., AUBRY T. et ALMEIDA F., 1997 - L'utilisation du quartz pendant la transition Gravettien/Solutréen au Portugal. Préhistoire, Anthropologie Méditerranéennes, 6, p. 289-303.

\section{NOTES}

1. Nous n'avons retrouvé, dans ce niveau, aucune lame aménagée par la technique de Kostienki.

2. cette torsion a déjà été décrite par T. Aubry et ses collaborateurs (1997).

\section{RÉSUMÉS}

Comme pour un certain nombre d'outils en silex du Paléolithique supérieur (pièces carénées, pièces esquillées) la possibilité de la fonction de nucléus pour les burins du Raysse n'a été soulevée que tardivement. Par l'étude technologique des burins du Raysse de la couche $\mathrm{V}$ du Flageolet I, cet article a pour objectif de contribuer à la clarification de cette question, importante en termes d'interprétation fonctionnelle de l'assemblage.

Like a number of other Upper Palaeolithic stone tools (carinate pieces, "pièces esquillées"), the possibility that the Raysse burins may have functionned as cores has lately been raised. With this technological study of the Raysse burins from the level V of Le Flageolet I, the objective of this 
article is to contribute to the clarification of this question, important in terms of functional interpretation of the assemblage.

\section{INDEX}

Mots-clés: Gravettien, burin du Raysse, burin de Bassaler, technologie lithique, production lamellaire, nucléus à lamelles, chute de burin

Keywords : Gravettian, Raysse burin, Bassaler burin, lithic technology, bladelet production, bladelet core, burin spall

\section{AUTEUR}

\section{GERALDINE LUCAS}

Institut de Préhistoire et de Géologie du Quaternaire, Université Bordeaux 1, UMR 5808, avenue des Facultés, 33405 Talence cedex, France. 